\title{
Asymmetric co-evaporated Co-Cr films: magnetic parameters and reversal mechanism
}

\author{
Herma van Kranenburg, Cock Lodder and Theo J.A. Popma \\ Mesa Research Institute, University of Twente, P.O. Box 217, 7500 AE Enschede, The Netherlands
}

\begin{abstract}
The magnetic parameters of co-evaporated $\mathrm{Co}-\mathrm{Cr}$ films, where $\mathrm{Cr}$ was deposited obliquely, are investigated. Process-induced compositional separation enhanced the energy product. When the substrate was rotated during evaporation, the perpendicular characteristics were lost.
\end{abstract}

\section{Introduction}

Co-evaporated $\mathrm{Co}-\mathrm{Cr}$ films were deposited with two e-beam sources. The Co vapour reached the substrate under normal incidence, and the $\mathrm{Cr}$ vapour at an incidence angle of $45-48^{\circ}$. The substrate was $\mathrm{Si}$ and the deposition was carried out at room temperature $\left(T_{\mathrm{R}}\right)$. The average $\mathrm{Cr}$ content was varied from 14 to 36 at\%; film thicknesses were $200-290 \mathrm{~nm}$. One series was made with non-rotating substrates (referred to as $\mathrm{L}+$ number); a second series was made with rotating substrates (referred to as LR + number), with rotation speeds of either 30 or $82 \mathrm{rpm}$. The total deposition rate was approximately $13 \AA / s$ (L24 and L25) or $9 \AA / s$ (rest). Typical background pressures were $2-4 \times 10^{-7}$ mbar.

The magnetic properties were characterized by VSM (hysteresis behaviour) and torque measurements (anisotropy constants). Average compositions were measured by XRF. The microstructure was investigated with SEM (columnar size) and XRD (texture and phase configuration). The growth of the (L-) films was simulated using a 2D Monte Carlo simulation program.

\section{Results}

With the two separate vapour flux directions of Co and $\mathrm{Cr}$, the resulting morphology was determined by the two angles of incidence and partial vapour rates. A weighted average of these yielded an effective incoming vapor direction. In the case of the L-samples the effective incoming direction was tilted slightly towards the $\mathrm{Cr}$ source. This effective incidence angle results in a tilted columnar structure; e.g., for sample L25F (see

Correspondence to: Dr. J.C. Lodder, Mesa Research Institute, University of Twente, P.O. Box 217, 7500 AE Enschede, the Netherlands. Tel.: +31-53-892750; telefax: +3153-309547; telex 44200; E-mail: LODDER@UTWENTE.NL. table 1) we measured a columnar tilt angle of around $10-11^{\circ}$ with respect to the film normal. In the case of the LR samples the tilt angle of the $\mathrm{Cr}$ vapour averaged to zero, and a perpendicular microstructure was observed. The structure of both $\mathrm{L}$ and $\mathrm{LR}$ samples was hcp with $(0002)$ lattice spacing $2.027 \AA$ ( $\leq 24$ at $\% \mathrm{Cr})$ or $2.030 \AA$ ( $\geq 26$ at $\% \mathrm{Cr})$. In all cases the $c$-axis was perpendicular to the substrate.

The effect of the oblique deposition of $\mathrm{Cr}$ is that shadowing plays a role in film formation. For the $\mathrm{L}$ samples $\mathrm{Cr}$ is most likely to impinge on one side of the columns at every stage of film growth. The low substrate temperature prohibits large adatom mobility and thus the $\mathrm{Cr}$ atoms will stick to a place near the impingement spot, resulting in local variations in $C o$ and $\mathrm{Cr}$, where $\mathrm{Cr}$ is enriched at one side of the columns: the process-induced compositional separation. This process-geometry induced effect has been found to exist in symmetrical co-evaporated $\mathrm{Co}-\mathrm{Cr}$ films [1], where it enhanced the energy product $\left(M_{\mathrm{s}} \times H_{\mathrm{c} \perp}\right)$. The shadowing concept is applied in a 2D Monte Carlo simulation of film growth. The programme includes surface relaxation by thermally induced surface diffusion and atomic attraction for a two-component system. For model interaction energies, a link to the real bonding energies of $\mathrm{Co}$ and $\mathrm{Cr}$ was made [2]. Figure 1 shows a simulation result for low diffusion, which can be compared with the $L$ samples (in a one-component system the degree of diffusion was comparable with a quotient of the substrate and melting temperature of 0.285). Clearly observable is a small tilt of the columnar-alikes towards the $\mathrm{Cr}$ source and the enhanced $\mathrm{Cr}$ distribution at one side. As in symmetrical coevaporated $\mathrm{Co}-\mathrm{Cr}$ films, this results in an enhanced energy product. Saturation magnetizations are much higher than those for homogeneous bulk $\mathrm{Co}-\mathrm{Cr}$ layers and coercivity values are moderate, decreasing with increasing $M_{\mathrm{s}}$. Figure 2 shows a typical saturation magnetization curve measured with VSM. Table 1 gives 
Table 1

Magnetic properties; samples LR19 and LR30 were rotated at $82 \mathrm{rpm}$, and LR31 and LR03 at $30 \mathrm{rpm}$

\begin{tabular}{|c|c|c|c|c|c|c|c|c|}
\hline Film & $\begin{array}{l}h \\
\text { [nm] }\end{array}$ & $\begin{array}{l}\mathrm{Cr} \\
\text { [at\%] }\end{array}$ & $\begin{array}{l}M_{\mathrm{s}} \\
{[\mathrm{kA} / \mathrm{m}]}\end{array}$ & $\begin{array}{l}H_{\mathrm{c} \perp} \\
{[\mathrm{kA} / \mathrm{m}]}\end{array}$ & $\begin{array}{l}H_{\mathrm{c} \|} \\
{[\mathrm{kA} / \mathrm{m}]}\end{array}$ & $\begin{array}{l}K_{1} \\
{\left[\mathrm{~kJ} \mathrm{~m}^{-3}\right]}\end{array}$ & $\begin{array}{l}K_{1} \\
{\left[\mathrm{~kJ} \mathrm{~m}^{-3}\right]}\end{array}$ & $\begin{array}{l}\gamma \\
\text { [deg] }\end{array}$ \\
\hline $\mathrm{L} .24 \mathrm{~F}$ & 284 & 13.8 & 772 & 31 & 21 & - & - & - \\
\hline L24E & 288 & 15.2 & 727 & 33 & 23 & 153 & 15 & 8 \\
\hline L24D & 289 & 16.3 & 681 & 38 & 24 & 187 & 22 & 6 \\
\hline L25F & 207 & 21.7 & 477 & 55 & 27 & 120 & 20 & 9 \\
\hline L.25E & 211 & 23.0 & 427 & 60 & 29 & 110 & 21 & 8 \\
\hline L.25D & 214 & 24.5 & 380 & 62 & 32 & 104 & 21 & 7 \\
\hline L05F & 198 & 28.6 & 285 & 50 & 28 & 50 & 15 & 9 \\
\hline L05E & 205 & 30.2 & 232 & 55 & 25 & 74 & 14 & 5 \\
\hline L05D & 213 & 32.7 & 198 & 61 & 24 & 68 & 14 & 5 \\
\hline LR19F & 196 & 26.8 & 361 & 13 & 10 & 124 & 0.7 & 0 \\
\hline LR19E & 204 & 26.0 & 342 & 12 & 10 & 112 & 0.3 & 0 \\
\hline LR30 & 207 & 20.8 & 503 & 18 & 20 & 138 & - & 0 \\
\hline LR31F & 205 & 17.6 & 612 & 23 & 22 & - & - & - \\
\hline LR31E & 207 & 18.4 & 613 & 22 & 22 & 146 & 0.6 & 0 \\
\hline LR03 & 199 & 35.6 & 63 & 5 & 8 & 14 & - & 0 \\
\hline
\end{tabular}

\section{a $q^{\text {co }}$}

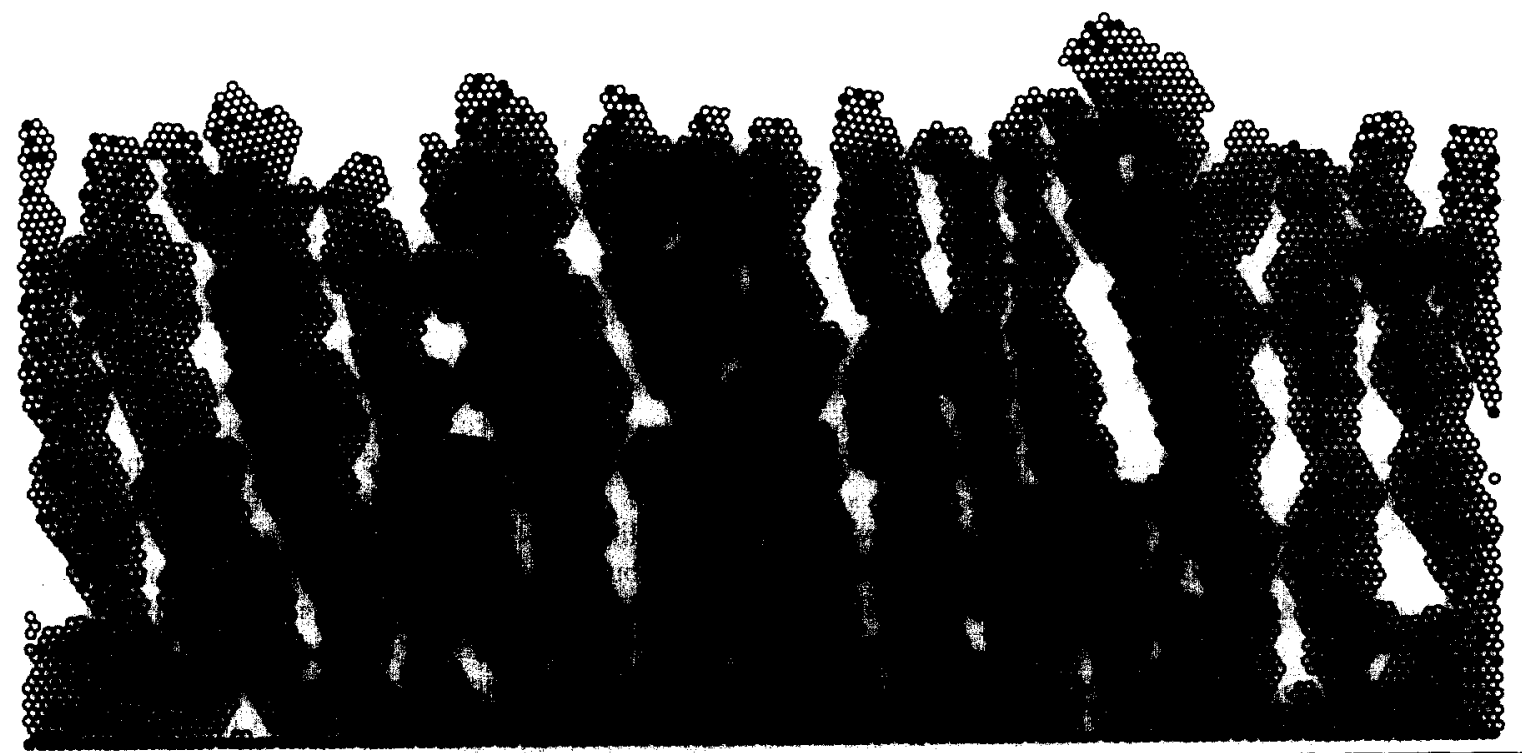

Fig. 1. Simulated microstructure with 22 at $\% \mathrm{Cr}$ and 78 at\% Co. The arrows above the figure indicate the vapour flux directions. 


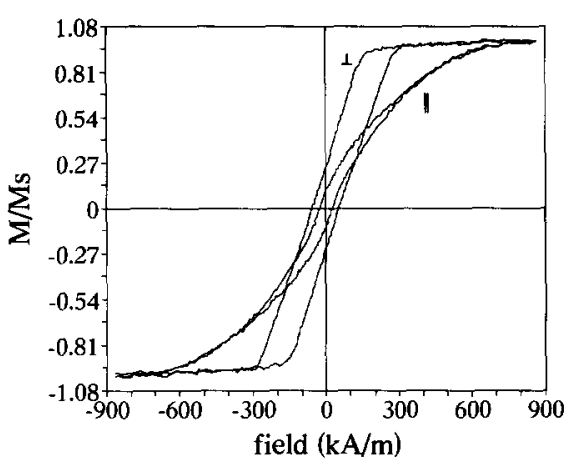

Fig. 2. Typical $M-H$ curves for the $\mathrm{L}$ samples (LM plane).

values of $M_{\mathrm{s}}, H_{\mathrm{c}}$ and the anisotropy constants for $\mathrm{L}$ films with various average compositions. $H_{\mathrm{c} \|}$ values are measured in the evaporation plane (the so-called LM plane). In the transversal in-plane direction (TM) $H_{\mathrm{c} \|}$ is slightly smaller. In the transversal direction there is a homogeneous supply of vapour material and no shadowing (no oblique incidence) occurs. This should result in a higher packing density, whereas the shadowing in the evaporation plane causes open regions. The discontinuities caused by the open regions hinder domain wall movement by acting as pinning centres. This raises the coercivity, so that a higher $H_{\mathrm{c} \|}$ in the evaporation plane with respect to the transversal direction is possible. From the hcp crystalline anisotropy the secondorder constant is smaller than $10 \%$ of the first-order constant. In table 1 we listed $K_{1}$ : a combination of hcp crystalline (oriented $\| c$-axis, which is $\perp$ substrate) and columnar shape anisotropy. $K_{1}$ is slightly tilted towards the $\mathrm{Cr}$ direction. Both the magnitude and tilt angle ( $\gamma$ : measured with respect to the film normal) of $K_{1}$ decrease with increasing $\mathrm{Cr}$ content. The $\mathrm{L}$ films also have an in-plane anisotropy constant in the transversal direction $K_{t}$ which is some $20 \%$ of $K_{1}$. Since the 'projection' of the oblique anisotropy constants in-plane is smaller than $K_{\mathrm{t}}$ the transversal direction is the effective in-plane easy axis direction. An effective quality factor would therefore be slightly smaller in the transversal than in the longitudinal direction. $K_{\mathrm{t}}$ may be caused by the shape anisotropy of aggregates of columns in the transversal direction. In high-resolution SEM observations we could not distinguish any (clear) aggregation due to the small columnar size $(<10 \mathrm{~nm})$.

The rotated samples (LR films) have totally different saturation magnetization curves from those of $\mathrm{L}$ films; large shoulders are present in the $M-H$ loops (see fig. 3). The shoulders are more pronounced in the faster rotated films, which is accompanied by a trend of decreasing $H_{c \perp}$. All LR films have low coercivities: $H_{\mathrm{c} \perp}=10-20 \mathrm{kA} / \mathrm{m}$. There are no longer differences

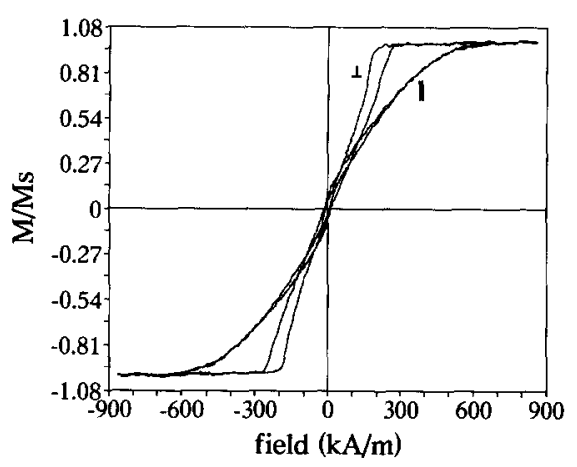

Fig. 3. Typical $M-H$ curves for the $\mathrm{LR}$ samples ( $\mathrm{LM}=\mathrm{TM}$ plane).

between $H_{\mathrm{cl}}$ in the LM and TM directions. The anisotropy constant $K_{1}$ is around $130 \mathrm{~kJ} / \mathrm{m}^{-3}$ and is perpendicular (see table 1). In-plane no preferred anisotropy axis is present (in-plane isotropic). The latter two facts are understandable: rotation during evaporation averages out all induced effects from the oblique $\mathrm{Cr}$ direction. During low rotation in every rotation cycle, an average of 4.9 atoms arrive at the film surface (and 7.2 in the high rotation). The problem for not obtaining a high $H_{c \perp}$ probably has its origin in the smaller discontinuities (less broad open regions and $\mathrm{Cr}$-rich regions). At every stage of film growth the $\mathrm{Cr}$ and $\mathrm{Co}$ arrive at the same height of film development. Preferably, the Co should be deposited before the $\mathrm{Cr}$, such that the Co columns form first, and the $\mathrm{Cr}$ then impinges on the boundaries rather than on the tops of the columns. The curvature of the columnar top is certainly important in this matter. In LR films the effective incoming vapour direction is perpendicular to the substrate so that the columnar tops are unlikely to be slanted in one direction, which will not encourage (process-induced) separation of $\mathrm{Cr}$ and $\mathrm{Co}$. The average columnar size $(<10 \mathrm{~nm})$ in LR films is smaller than that of $L$ films.

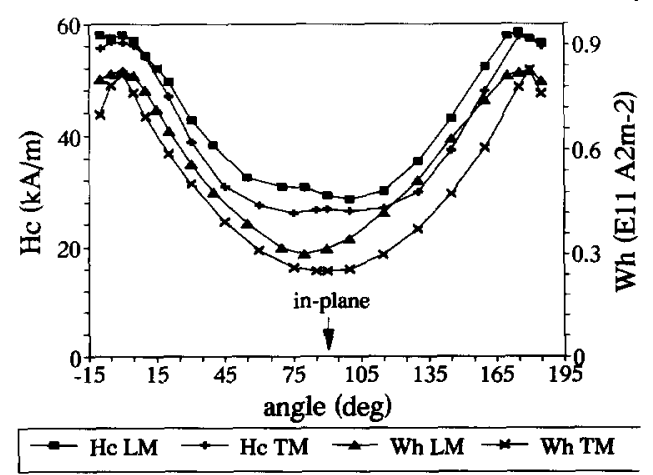

Fig. 4. Angle dependence of $H_{\mathrm{c}}$ and $W_{\mathrm{h}}$ for L25E. 


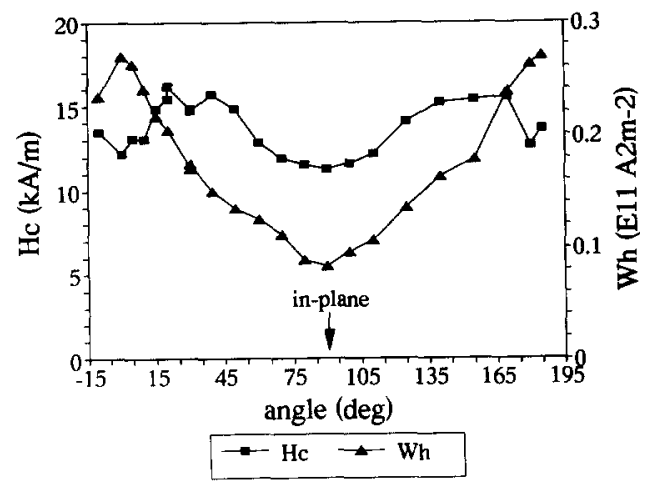

Fig. 5. Angle dependence of $H_{\mathrm{c}}$ and $W_{\mathrm{h}}$ for LR19E.

The angle-dependent coercivity and hysteresis loss $W_{\mathrm{h}}$ are given in figs. 4 and 5 for a L and LR films, respectively. A proper interpretation of our-of-plane coercivity measurements requires demagnetization corrections using a biaxial VSM [3]. We were unable to perform such measurements, so we give only a very qualitative interpretation of the (uncorrected) curves of figs. 4 and 5. The strong decrease in both $H_{\mathrm{c}}$ and $W_{\mathrm{h}}$ towards the in-plane direction (angle $90^{\circ}$ in fig. 4) for the $\mathrm{L}$ film indicates that considerable domain wall pinning occurs. Reversal of the magnetization is most likely to proceed through either rotation or domain wall movement where the displacement of domain walls is largely hindered by the open regions in between the columns and by chemical inhomogeneities, i.e. the $\mathrm{Cr}$ rich regions formed by the process-induced compostional separation. The LR films show different behaviour. The coercivity is not a straightforward decreasing function of the angle, but has a local maximum around $20-40^{\circ}$ from the film normal. The hysteresis loss also does not decrease as steeply, indicating that in LR films domain wall displacement is likely to dominate.

\section{Conclusions}

Co-evaporated $\mathrm{Co}-\mathrm{Cr}$ films, where $\mathrm{Cr}$ was deposited obliquely and the substrate was not rotated, show enhanced perpendicular characteristics due to process-induced compositional separation. When the substrate was rotated during evaporation the distribution of $\mathrm{Cr}$ and $\mathrm{Co}$ was more homogeneous and the perpendicular characteristics were lost.

\section{References}

[1] H. van Kranenburg, J.C. Lodder, Th.J.A. Popma, K. Takei and Y. Maeda, J. Magn. Soc. Jpn. 15 Suppl. S2 (1991) 33.

[2] S. Müller-Pfeiffer, H. van Kranenburg and J.C. Lodder, Thin Solid Films 213 (1992) 143.

[3] J.P.C. Bernards and H.A.J. Cramer, IEEE Trans. Magn. MAG-27 (1991) 4873. 